\title{
STOCHASTIC MAXIMUM PRINCIPLE FOR OPTIMAL CONTROL PROBLEM OF FORWARD AND BACKWARD SYSTEM
}

\author{
WENSHENG XU ${ }^{1}$
}

(Received 19 July 1993; revised 28th March 1994)

\begin{abstract}
The maximum principle for optimal control problems of stochastic systems consisting of forward and backward state variables is proved, under the assumption that the diffusion coefficient does not contain the control variable, but the control domain need not be convex.
\end{abstract}

\section{Introduction}

The stochastic optimal control problem is important in control theory. A lot of work has been done on the forward stochastic system. See, for example, Ahmed [2], Bensoussan [5], Cadenillas and Karatzas [7], Elliott [8], H. J. Kushner [10], Peng [12]. Recently, another kind of stochastic system, the forward and backward stochastic system, has been developed and studied for its applications in the financial market. In [13], Peng studied the optimal control problem of such a system. The maximum principle he obtained is in local form.

In this paper, we discuss a simplified problem of one in [13], in which the diffusion coefficient does not contain control. We use the "spike variation" method to derive the maximum principle in global form. Thus the control domain is not necessarily convex. For the case when there are initial state constraints and final state constraints, we also obtain a global result by using Ekeland's variational principle. Since some financial models are in the form of forward and backward stochastic systems, our results may have applications in the financial market.

Since the existence problem of optimal control is a different issue, we do not incorporate it in this paper. Some results in this field can be seen in Ahmed [1], [2].

This paper is organized as follows. In Section 2, we state the problem and our main assumptions. In Section 3, we study the variational equations and variational inequality. In Section 4, we obtain the maximum principle in global form. In the last

'Department of Mathematics, Zhejiang University, Hongzhou 310027, China.

(C) Australian Mathematical Society, 1995, Serial-fee code 0334-2700/95 
section, we show how to obtain the maximum principle in case initial and final state contraints are imposed.

\section{Statement of the problem}

Let $(\Omega, \mathscr{F}, P)$ be a probability space with filtration $\mathscr{F}^{\prime}$ and $W(\cdot)$ a $R^{d}$-valued standard Wiener process. We assume $\mathscr{F} t=\sigma\{W(s): 0 \leq s \leq t\}$. We consider the following forward and backward stochastic control system:

$$
\left\{\begin{aligned}
d x & =f(x, v, t) d t+\sigma(x, t) d W_{t} \\
x(0) & =x_{0} \\
d y & =g(x, y, z, v, t) d t+z d W_{t} \\
y(T) & =h(x(T))
\end{aligned}\right.
$$

where

$$
\begin{aligned}
f: R^{n} \times R^{k} \times[0, T] \rightarrow R^{n}, \\
\sigma: R^{n} \times[0, T] \rightarrow \mathscr{L}\left(R^{d} ; R^{n}\right), \\
g: R^{n} \times R^{m} \times \mathscr{L}\left(R^{d} ; R^{m}\right) \times R^{k} \times[0, T] \rightarrow R^{m}, \\
h: R^{n} \rightarrow R^{m} .
\end{aligned}
$$

Let $U$ be a non-empty subset of $R^{k}$. We set

$$
\mathscr{U}_{a d}=\left\{v(\cdot) \in \mathscr{L}_{\mathscr{F}}^{2}\left(0, T ; R^{k}\right): v(t) \in U, \text { a.e., a.s. }\right\}
$$

Our optimal control problem is to minimize the cost function

$$
J(v(\cdot))=E \gamma(y(0))
$$

over $\mathscr{U}_{a d}$, where $\gamma: R^{m} \rightarrow R^{1}$.

We assume:

$\left(H_{1}\right) f, g, \sigma, h, \gamma$ are continuously differentiable with respect to $(x, y, z)$;

$\left(H_{2}\right)$ the derivatives of $f, g$ and $\sigma$ with respect to $x, y, z$ are bounded,

$$
\left|f_{x}\right| \leq C, \quad \text { for } \quad f_{x}=f_{x}, \sigma_{x}, g_{x}, g_{y}, g_{z},
$$

and

$$
\left|h_{x}\right| \leq C(1+|x|), \quad\left|\gamma_{y}\right| \leq C(1+|y|) .
$$




\section{Variational equations and variational inequality}

The purpose of this section is to introduce the usual first order variational equations and to derive variational inequality. Let $(u(\cdot), x(\cdot), y(\cdot), z(\cdot))$ be an optimal solution of the problem. We introduce the following spike variational control:

$$
u^{\epsilon}(t)= \begin{cases}v, & \tau \leq t \leq \tau+\epsilon \\ u(t), & \text { otherwise }\end{cases}
$$

where $\epsilon>0$ is sufficiently small, $v$ is an arbitrary $\mathscr{F}^{\tau}$-measurable random variable with values in $U, 0 \leq t<T$, and $\sup _{\omega \in \Omega}|v(\omega)|<\infty$. Let $\left(x^{\epsilon}(\cdot), y^{\epsilon}(\cdot), z^{\epsilon}(\cdot)\right)$ be the trajectory of system (1) corresponding to control $u^{\epsilon}(\cdot)$.

We introduce the following variational equations:

$$
\left\{\begin{aligned}
d x_{1} & =\left[f_{x} x_{1}+f\left(u^{\epsilon}\right)-f(u)\right] d t+\sigma_{x} x_{1} d W_{t}, \\
x_{1}(0) & =0 \\
d y_{1} & =\left[g_{x} x_{1}+g_{y} y_{1}+g_{z} z_{1}+g\left(u^{\epsilon}\right)-g(u)\right] d t+z_{1} d W_{t}, \\
y_{1}(T) & =h_{x}(x(T)) x_{1}(T) .
\end{aligned}\right.
$$

For convenience, we use the following notation in this paper.

$$
\begin{aligned}
f_{x} & \triangleq f_{x}(x(t), u(t), t), & g_{x} & \triangleq g_{x}(x(t), y(t), z(t), u(t), t), \\
f\left(u^{\epsilon}\right) & \triangleq f\left(x(t), u^{\epsilon}(t), t\right), & f(t) & \triangleq f(x(t), u(t), t), \quad \text { etc. }
\end{aligned}
$$

The variational inequality can be obtained from the fact $J\left(u^{\epsilon}(\cdot)\right)-J(u(\cdot)) \geq 0$. The following lemmas are needed to establish the inequality.

LemMA 1. Suppose $\left(H_{1}\right)$ and $\left(H_{2}\right)$ hold. For the first order variations $x_{1}, y_{1}, z_{1}$, we have the following estimations:

$$
\begin{gathered}
\sup _{0 \leq t \leq T} E\left|x_{1}(t)\right|^{2} \leq C \epsilon^{2}, \\
\sup _{0 \leq t \leq T} E\left|x_{1}(t)\right|^{4} \leq C \epsilon^{4}, \\
\sup _{0 \leq t \leq T} E\left|y_{1}(t)\right|^{2} \leq C \epsilon^{2}, \\
\sup _{0 \leq t \leq T} E\left|y_{1}(t)\right|^{4} \leq C \epsilon^{4}, \\
E \int_{0}^{T}\left(z_{1}(s)\right)^{2} d s \leq C \epsilon^{2}, \\
E\left(\int_{0}^{T}\left(z_{1}(s)\right)^{2} d s\right)^{2} \leq C \epsilon^{4} .
\end{gathered}
$$


[4] Stochastic maximum principle for optimal control problem of forward and backward system

ProOF. We first prove (3) and (4). The first equation of (2) yields

$$
\begin{aligned}
& E\left|x_{1}(t)\right|^{2}=E\left(\int_{0}^{t}\left[f_{x} x_{1}+f\left(u^{\epsilon}\right)-f(u)\right] d s+\int_{0}^{t} \sigma_{x} x_{1} d W_{s}\right)^{2} \\
& \leq 3\left[E\left(\int_{0}^{t} f_{x} x_{1} d s\right)^{2}+E\left(\int_{0}^{t}\left[f\left(u^{\epsilon}\right)-f(u)\right] d s\right)^{2}+E \int_{0}^{t}\left(\sigma_{x} x_{1}\right)^{2} d s\right] \\
& \leq 6 C^{2} T E \int_{0}^{t} x_{1}{ }^{2} d s+3 E\left(\int_{0}^{t}\left(f\left(u^{\epsilon}\right)-f(u)\right) d s\right)^{2} .
\end{aligned}
$$

Applying Gronwall's inequality,

$$
E\left|x_{1}(t)\right|^{2} \leq C \epsilon^{2}, \quad \text { for } t \in[0, T] \text { uniformly. }
$$

Similarly (4) holds.

We next estimate $y_{1}$ and $z_{1}$. Squaring both sides of

$$
\begin{aligned}
& -y_{1}(t)-\int_{t}^{T} z_{1}(s) d W_{s} \\
= & -h_{x}(x(T)) x_{1}(T)+\int_{t}^{T}\left(g_{x} x_{1}+g_{y} y_{1}+g_{z} z_{1}+g\left(u^{\epsilon}\right)-g(u)\right) d s,
\end{aligned}
$$

and using the fact that

$$
E y_{1}(t) \int_{t}^{T} z_{1}(s) d W_{s}=0
$$

we get

$$
\begin{aligned}
E\left|y_{1}(t)\right|^{2}+E \int_{t}^{T}\left(z_{1}(s)\right)^{2} d s \\
=E\left(-h_{x}(x(T)) x_{1}(T)+\int_{t}^{T}\left(g_{x} x_{1}+g_{y} y_{1}+g_{z} z_{1}+g\left(u^{\epsilon}\right)-g(u)\right) d s\right)^{2} \\
\leq 5 C^{2} E x_{1}^{2}(T)+5 C^{2} T E \int_{t}^{T} x_{1}^{2}(s) d s+5 C^{2} T E \int_{t}^{T} y_{1}^{2}(s) d s \\
\quad+5 C^{2}(T-t) E \int_{t}^{T} z_{1}^{2}(s) d s+5 E\left(\int_{t}^{T}\left(g\left(u^{\epsilon}\right)-g(u)\right) d s\right)^{2}
\end{aligned}
$$

Thus

$$
\begin{aligned}
E\left|y_{1}(t)\right|^{2}+\frac{1}{2} E \int_{t}^{T} z_{1}^{2}(s) d s \leq & 5 C^{2} E x_{1}^{2}(T)+5 C^{2} T E \int_{0}^{T} x_{1}^{2}(s) d s \\
& +5 C^{2} T E \int_{t}^{T} y_{1}^{2}(s) d s+5 E\left(\int_{t}^{T}\left(g\left(u^{\epsilon}\right)-g(u)\right) d s\right)^{2},
\end{aligned}
$$


with $\delta=\frac{1}{10 c^{2}}, t \in[T-\delta, T]$.

Applying Gronwall's inequality,

$$
\begin{gathered}
E\left|y_{1}(t)\right|^{2} \leq C \epsilon^{2}, \quad t \in[T-\delta, T], \\
E \int_{t}^{T} z_{1}^{2}(s) d s \leq C \epsilon^{2}, \quad t \in[T-\delta, T] .
\end{gathered}
$$

Similarly we have

$$
\begin{aligned}
-y_{1}(t) & -\int_{t}^{T-\delta} z_{1}(s) d W_{s} \\
= & -Y_{1}(T-\delta)+\int_{t}^{T-\delta}\left(g_{x} x_{1}+g_{y} y_{1}+g_{z} z_{1}+g\left(u^{\epsilon}\right)-g(u)\right) d s .
\end{aligned}
$$

So

$$
\begin{aligned}
E\left|y_{1}(t)\right|^{2}+E \int_{t}^{T-\delta} z_{1}^{2}(s) d s \leq & 5 E\left|y_{1}(T-\delta)\right|^{2}+5 C^{2} T E \int_{t}^{T-\delta} x_{1}^{2}(s) d s \\
& +5 C^{2} T E \int_{t}^{T-\delta} y_{1}^{2}(s) d s \\
& +5 C^{2}(T-\delta-t) E \int_{t}^{T-\delta} z_{1}^{2}(s) d s \\
& +5 E\left(\int_{t}^{T-\delta}\left(g\left(u^{\epsilon}\right)-g(u)\right) d s\right)^{2} .
\end{aligned}
$$

Thus

$$
\begin{gathered}
E\left|y_{1}(t)\right|^{2} \leq C \epsilon^{2}, \quad t \in[T-2 \delta, T], \\
E \int_{t}^{T-\delta} z_{1}^{2}(s) d s \leq C \epsilon^{2}, \quad t \in[T-2 \delta, T] .
\end{gathered}
$$

After a finite number of iterations, (5) and (7) are obtained. (6) and (8) can be proved by using a similar method and the inequality

$$
E\left(\int_{t}^{T} z_{1}(s) d W_{s}\right)^{4} \geq \beta E\left(\int_{t}^{T} z_{1}^{2}(s) d s\right)^{2}, \quad \beta>0 .
$$

LEMMA 2. Suppose $\left(H_{1}\right)$ and $\left(H_{2}\right)$ hold. Then we have the following estimations:

$$
\sup _{0 \leq t \leq T} E\left|x^{\epsilon}(t)-x(t)-x_{1}(t)\right|^{2} \leq C_{\epsilon} \epsilon^{2}, \quad C_{\epsilon} \rightarrow 0,
$$


[6] Stochastic maximum principle for optimal control problem of forward and backward system

$$
\begin{array}{ll}
\sup _{0 \leq t \leq T} E\left|y^{\epsilon}(t)-y(t)-y_{1}(t)\right|^{2} \leq C_{\epsilon} \epsilon^{2}, & C_{\epsilon} \rightarrow 0, \\
E \int_{0}^{T}\left|z^{\epsilon}(t)-z(t)-z_{1}(t)\right|^{2} d s \leq C_{\epsilon} \epsilon^{2} & C_{\epsilon} \rightarrow 0 .
\end{array}
$$

PROOF. To prove (9), we observe that

$$
\begin{aligned}
& \int_{0}^{t} f\left(x+x_{1}, u^{\epsilon}\right) d s+\int_{0}^{t} \sigma\left(x+x_{1}\right) d W_{s} \\
&=\int_{0}^{t}\left[f\left(x, u^{\epsilon}\right)\right.\left.+\int_{0}^{1} f_{x}\left(x+\lambda x_{1}, u^{\epsilon}\right) d \lambda x_{1}\right] d s \\
&+\int_{0}^{t}\left[\sigma(x)+\int_{0}^{1} \sigma_{x}\left(x+\lambda x_{1}\right) d \lambda x_{1}\right] d W_{s} \\
&=\int_{0}^{t} f(x, u) d s+\int_{0}^{t} \sigma(x) d W_{s}+\int_{0}^{t}\left[f_{x} x_{1}+f\left(u^{\epsilon}\right)-f(u)\right] d s \\
& \quad+\int_{0}^{t} \sigma_{x} x_{1} d W_{s}+\int_{0}^{t} A^{\epsilon} d s+\int_{0}^{t} B^{\epsilon} d W_{s} \\
&=x(t)-x_{0}+x_{1}(t)+\int_{0}^{t} A^{\epsilon} d s+\int_{0}^{t} B^{\epsilon} d W_{s}
\end{aligned}
$$

in which

$$
\begin{aligned}
& A^{\epsilon}=\int_{0}^{1}\left[f_{x}\left(x+\lambda x_{1}, u^{\epsilon}\right)-f_{x}(x, u)\right] d \lambda x_{1}, \\
& B^{\epsilon}=\int_{0}^{1}\left[\sigma_{x}\left(x+\lambda x_{1}\right)-\sigma_{x}(x)\right] d \lambda x_{1} .
\end{aligned}
$$

It follows easily from Lemma 1 that

$$
\sup _{0 \leq t \leq T} E\left\{\left(\int_{0}^{t} A^{\epsilon} d s\right)^{2}+\left(\int_{0}^{t} B^{\epsilon} d W_{s}\right)^{2}\right\}=\mathrm{o}\left(\epsilon^{2}\right) .
$$

Since

$$
x^{\epsilon}(t)-x_{0}=\int_{0}^{t} f\left(x^{\epsilon}, u^{\epsilon}\right) d s+\int_{0}^{t} \sigma\left(x^{\epsilon}\right) d W_{s}
$$

we get

$$
\begin{aligned}
x^{\epsilon}(t)-x(t)-x_{1}(t)= & \int_{0}^{t} C^{\epsilon}(s)\left(x^{\epsilon}-x-x_{1}\right) d s+\int_{0}^{t} D^{\epsilon}(s)\left(x^{\epsilon}-x-x_{1}\right) d W_{s} \\
& +\int_{0}^{t} A^{\epsilon} d s+\int_{0}^{t} B^{\epsilon} d W_{s}
\end{aligned}
$$


with

$$
\begin{aligned}
& C^{\epsilon}(s)=\int_{0}^{1} f_{x}\left(x+x_{1}+\lambda\left(x^{\epsilon}-x-x_{1}\right), u^{\epsilon}\right) d \lambda, \\
& D^{\epsilon}(s)=\int_{0}^{1} \sigma_{x}\left(x+x_{1}+\lambda\left(x^{\epsilon}-x-x_{1}\right), u^{\epsilon}\right) d \lambda .
\end{aligned}
$$

Using Gronwall's inequality, (9) follows from the above relation and (12).

We next prove (10) and (11). It can be easily checked that

$$
\begin{array}{r}
\int_{t}^{T} g\left(x+x_{1}, y+y_{1}, z+z_{1}, u^{\epsilon}\right) d s+\int_{t}^{T}\left(z(s)+z_{1}(s)\right) d W_{s} \\
=h(x(T))+h_{x}(x(T)) x_{1}(T)-y(t)-y_{1}(t)+\int_{t}^{T} G^{\epsilon} d s
\end{array}
$$

where

$$
\begin{aligned}
G^{\epsilon}= & \int_{0}^{1}\left(g_{x}\left(x+\lambda x_{1}, y+\lambda y_{1}, z+\lambda z_{1}, u^{\epsilon}\right)-g_{x}\right) d \lambda x_{1} \\
& +\int_{0}^{1}\left(g_{y}\left(x+\lambda x_{1}, y+\lambda y_{1}, z+\lambda z_{1}, u^{\epsilon}\right)-g_{y}\right) d \lambda y_{1} \\
& +\int_{0}^{1}\left(g_{z}\left(x+\lambda x_{1}, y+\lambda y_{1}, z+\lambda z_{1}, u^{\epsilon}\right)-g_{z}\right) d \lambda z_{1} .
\end{aligned}
$$

So we have

$$
\begin{aligned}
-\left(y^{\epsilon}(t)-y(t)-y_{1}(t)\right)= & -\left(h\left(x^{\epsilon}(T)\right)-h(x(T))\right)+h_{x}(x(T)) x_{1}(T) \\
& +\int_{t}^{T}\left[g\left(x^{\epsilon}, y^{\epsilon}, z^{\epsilon}, u^{\epsilon}\right)-g\left(x+x_{1}, y+y_{1}, z+z_{1}, u^{\epsilon}\right)\right] d s \\
& +\int_{t}^{T}\left(z^{\epsilon}(s)-z(s)-z_{1}(s)\right) d W_{s}+\int_{1}^{T} G^{\epsilon} d s .
\end{aligned}
$$

Thus it follows that

$$
\begin{aligned}
E\left|y^{\epsilon}(t)-y(t)-y_{1}(t)\right|^{2}+E \int_{t}^{T}\left|z^{\epsilon}(s)-z(s)-z_{1}(s)\right|^{2} d s \\
=E\left\{-\left(h\left(x^{\epsilon}(T)\right)-h\left(x(T)+x_{1}(T)\right)\right)\right. \\
\quad-\int_{0}^{1}\left[h_{x}\left(x(T)+\lambda x_{1}(T)\right)-h_{x}(x(T))\right] d \lambda x_{1}(T)+\int_{t}^{T} G^{\epsilon} d s \\
\left.\quad+\int_{1}^{T}\left[g\left(x^{\epsilon}, y^{\epsilon}, z^{\epsilon}, u^{\epsilon}\right)-g\left(x+x_{1}, y+y_{1}, z+z_{1}, u^{\epsilon}\right)\right] d s\right\}^{2} .
\end{aligned}
$$


[8] Stochastic maximum principle for optimal control problem of forward and backward system

From Lemma 1 and (9), we see that

$$
\begin{aligned}
& \sup _{0 \leq t \leq T} E\left(\int_{t}^{T} G^{\epsilon} d s\right)^{2}=\mathrm{o}\left(\epsilon^{2}\right), \\
& E\left[h\left(x^{\epsilon}(T)\right)-h\left(x(T)+x_{1}(T)\right)\right]^{2}=\mathrm{o}\left(\epsilon^{2}\right) .
\end{aligned}
$$

We get (10) and (11) by applying the iterative method used in Lemma 1 to the above relation.

LEMMA 3. Under the assumptions $\left(H_{1}\right)$ and $\left(H_{2}\right)$, the following variational inequality holds:

$$
E \gamma_{y}(y(0)) y_{1}(0) \geq o(\epsilon)
$$

ProOF. From Lemma 2, we have the estimation

$$
E\left[\gamma\left(y^{\epsilon}(0)\right)-\gamma\left(y(0)+y_{1}(0)\right)\right]=o(\epsilon) .
$$

Therefore

$$
\begin{aligned}
0 & \leq E\left[\gamma\left(y(0)+y_{1}(0)\right)-\gamma(y(0))\right]+o(\epsilon) \\
& =E \gamma_{y}(y(0)) y_{1}(0)+o(\epsilon) .
\end{aligned}
$$

\section{The maximum principle in global form}

We introduce the adjoint equations and the Hamilton function for our problem. From the variational inequality obtained in Lemma 3, the maximum principle can be proved by applying Itô's formula.

The adjoint equations are

$$
\left\{\begin{aligned}
-d p & =\left(f_{x}^{*} p+g_{x}^{*} q+\sigma_{x}^{*} k\right) d t-k d W_{t} \\
p(T) & =-h_{x}^{*}(x(T)) q(T) \\
-d q & =g_{y}^{*} q d t+g_{z}^{*} q d W_{t} \\
q(0) & =-\gamma_{y}(y(0))
\end{aligned}\right.
$$

and the Hamiltonian function is

$$
H(x, y, z, v, p, q, k, t) \triangleq\langle p, f(x, v, t)\rangle+\langle q, g(x, y, z, v, t)\rangle+\langle k, \sigma(x)\rangle,
$$

where

$$
\begin{aligned}
H: & R^{n} \times R^{m} \times \mathscr{L}\left(R^{d} ; R^{m}\right) \times R^{k} \times R^{n} \times R^{m} \times \mathscr{L}\left(R^{d} ; R^{n}\right) \\
& \times[0, T] \rightarrow R^{n} .
\end{aligned}
$$


Relations (13) can be rewritten as

$$
\left\{\begin{aligned}
-d p & =H_{x} d t-k d W_{t} \\
p(T) & =-h_{x}^{*}(x(T)) q(T) \\
-d q & =H_{y} d t+H_{z} d W_{t} \\
q(0) & =-\gamma_{y}(y(0))
\end{aligned}\right.
$$

From (14) and Lemma 3, we have

THEOREM 1. Suppose $\left(H_{1}\right)$ and $\left(H_{2}\right)$ hold. Let $(u(\cdot), x(\cdot), y(\cdot), z(\cdot))$ be an optimal control and its corresponding trajectory of $(1),(p(\cdot), q(\cdot), k(\cdot))$ be the corresponding solution of (14). Then the maximum principle holds, that is

$$
\begin{aligned}
& H(x(t), y(t), z(t), v, p(t), q(t), k(t), t) \\
& \quad \geq H(x(t), y(t), z(t), u(t), p(t), q(t), k(t), t), \forall v \in U, \text { a.e., a.s. }
\end{aligned}
$$

PROOF. By applying Itô's formula to $\left\langle p, x_{1}\right\rangle$ and $\left\langle q, y_{1}\right\rangle$, it follows from (2) and (14) that

$$
\begin{aligned}
o(\epsilon) \leq E \gamma_{y}(y(0)) y_{1}(0) \\
=E \int_{0}^{T}\left[H\left(x(t), y(t), z(t), u^{\epsilon}(t), p(t), q(t), k(t)\right)\right. \\
\quad-H(x(t), y(t), z(t), u(t), p(t), q(t), k(t)] d t .
\end{aligned}
$$

From the above inequality, (15) can be easily derived.

\section{Problem with state constraints}

In this section, we discuss briefly the case when there are initial state constraints and final state constraints on the state variables:

$$
\begin{gathered}
E G_{1}(x(T))=0, \\
E G_{0}(y(0))=0,
\end{gathered}
$$

where

$$
\begin{array}{lll}
G_{1}: & R^{n} \rightarrow R^{n_{1}}, & n_{1}<n, \\
G_{0}: & R^{m} \rightarrow R^{m_{1}}, & m_{1}<m .
\end{array}
$$

We assume

$\left(H_{3}\right) G_{0}, G_{1}$ are continuously differentiable and $G_{0 x}, G_{1 x}$ are bounded;

$\left(H_{4}\right)$ the control domain $U$ is closed. 
[10] Stochastic maximum principle for optimal control problem of forward and backward system 181

We apply Ekeland's variational principle to solve this optimal control problem. We first define the metric in $\mathscr{U}_{a d}$. For $u(\cdot), v(\cdot) \in \mathscr{U}_{a d}$, let

$$
d(u(\cdot), v(\cdot))=E \operatorname{mes}\{t \in[0, T]: u(t) \neq v(t)\} .
$$

With this metric, $\left(\mathscr{U}_{a d}, d(\cdot, \cdot)\right)$ is a complete space.

Let $(u(\cdot), x(\cdot), y(\cdot), z(\cdot))$ be an optimal solution of the problem. For $v(\cdot) \in \mathscr{U}_{a d}$, we define

$$
\begin{aligned}
J_{\rho}(v(\cdot))= & \left\{E\left|G_{1}(x(T ; v))\right|^{2}+E\left|G_{0}(y(0 ; v))\right|^{2}\right. \\
& \left.+[E \gamma(y(0 ; v))-E \gamma(y(0))+\rho]^{2}\right\}^{\frac{1}{2}} .
\end{aligned}
$$

It can be checked that $J_{\rho}(v(\cdot)): \mathscr{U}_{a d} \rightarrow R^{1}$ is continuous, and

$$
\begin{aligned}
& J_{\rho}(v(\cdot)) \geq 0, \quad \forall v(\cdot) \in \mathscr{U}_{a d}, \\
& J_{\rho}(u(\cdot))=\rho .
\end{aligned}
$$

Obviously, we have

$$
J_{\rho}(u(\cdot)) \leq \inf _{v(\cdot) \in \mathscr{U}_{a d}} J_{\rho}(v(\cdot))+\rho .
$$

From Ekeland's variational principle, there exists $v_{\rho}(\cdot) \in \mathscr{U}_{a d}$ such that

(1) $J_{\rho}\left(v_{\rho}(\cdot)\right) \leq J_{\rho}(u(\cdot))=\rho$,

(2) $d\left(v_{\rho}(\cdot), u(\cdot)\right) \leq \sqrt{\rho}$,

(3) $J_{\rho}(w(\cdot)) \geq J_{\rho}\left(v_{\rho}(\cdot)\right)-\sqrt{\rho} d\left(w(\cdot), v_{\rho}(\cdot)\right), \quad$ for $w(\cdot) \in \mathscr{U}_{a d}$.

Making "spike variation"

$$
v_{\rho}^{\epsilon}(t)= \begin{cases}v, & \tau \leq t \leq \tau+\epsilon \\ v_{\rho}(t), & \text { otherwise }\end{cases}
$$

for $v_{\rho}$ as in Section 3, it follows from (16) that

$$
J_{\rho}\left(v_{\rho}^{\epsilon}(\cdot)\right)-J_{\rho}\left(v_{\rho}(\cdot)\right)+\sqrt{\rho} d\left(v_{\rho}^{\epsilon}(\cdot) ; v_{\rho}(\cdot)\right) \geq 0 .
$$

Let $\left(x_{\rho}, y_{\rho}, z_{\rho}\right)$ and $\left(x_{\rho}^{\epsilon}, y_{\rho}^{\epsilon}, z_{\rho}^{\epsilon}(\cdot)\right)$ be the corresponding trajectories to $v_{\rho}(\cdot)$ and $v_{\rho}^{\epsilon}(\cdot)$ respectively. The variational equations is the same as the one in Section 3, with $(x(\cdot)$, $y(\cdot), z(\cdot))=\left(x_{\rho}(\cdot), y_{\rho}(\cdot), z_{\rho}(\cdot)\right), u(\cdot)=v_{\rho}(\cdot)$. Similarly to the approach in Lemma 2 , it can be shown that

$$
\sup _{0 \leq t \leq T} E\left|x_{\rho}^{\epsilon}(t)-x_{\rho}(t)-x_{\rho 1}(t)\right|^{2} \leq C_{\epsilon} \epsilon^{2}, \quad C_{\epsilon} \rightarrow 0,
$$




$$
\begin{array}{ll}
\sup _{0 \leq i \leq T} E\left|y_{\rho}^{\epsilon}(t)-y_{\rho}(t)-y_{\rho 1}(t)\right|^{2} \leq C_{\epsilon} \epsilon^{2}, & C_{\epsilon} \rightarrow 0 \\
E \int_{0}^{T}\left|z_{\rho}^{\epsilon}(t)-z_{\rho}(t)-z_{\rho 1}(t)\right|^{2} \leq C_{\epsilon} \epsilon^{2}, & C_{\epsilon} \rightarrow 0 .
\end{array}
$$

Thus

$$
\begin{aligned}
J_{\rho}^{2}\left(v_{\rho}^{\epsilon}(\cdot)\right)-J_{\rho}^{2}\left(v_{\rho}(\cdot)\right)= & 2\left\langle E G_{1}\left(x_{\rho}(T)\right), E G_{1 x}\left(x_{\rho}(T)\right) x_{\rho 1}(T)\right\rangle \\
& +2\left\langle E G_{0}\left(y_{\rho}(0)\right), E G_{0 x}\left(y_{\rho}(0)\right) y_{\rho 1}(0)\right\rangle \\
& +2\left\langle E\left(\gamma\left(y_{\rho}(0)\right)-\gamma(y(0))+\rho\right), E \gamma_{y}\left(y_{\rho}(0)\right) y_{\rho 1}(0)\right\rangle+o(\epsilon)
\end{aligned}
$$

Let

$$
\begin{aligned}
h_{\rho 1}^{\epsilon} & =\frac{2 E G_{1}\left(x_{\rho}(T)\right)}{J_{\rho}\left(v_{\rho}^{\epsilon}(\cdot)\right)+J_{\rho}\left(v_{\rho}(\cdot)\right)}, \\
h_{\rho 2}^{\epsilon} & =\frac{2 E G_{0}\left(y_{\rho}(0)\right)}{J_{\rho}\left(v_{\rho}^{\epsilon}(\cdot)\right)+J_{\rho}\left(v_{\rho}(\cdot)\right)}, \\
h_{\rho 0}^{\epsilon} & =\frac{2 E\left[\gamma\left(y_{\rho}(0)\right)-\gamma(y(0))+\rho\right]}{J_{\rho}\left(v_{\rho}^{\epsilon}(\cdot)\right)+J_{\rho}\left(v_{\rho}(\cdot)\right)} .
\end{aligned}
$$

Since

$$
J_{\rho}(v(\cdot))>0, \quad J_{\rho}\left(v_{\rho}^{\epsilon}(\cdot)\right)>0, \quad J_{\rho}\left(v_{\rho}^{\epsilon}(\cdot)\right) \rightarrow J_{\rho}\left(v_{\rho}(\cdot)\right), \quad \epsilon \rightarrow 0
$$

it follows from (17) and (18) that

$$
\begin{aligned}
\left\langle h_{\rho 1}^{\epsilon},\right. & \left.E G_{1 x}\left(x_{\rho}(T)\right) x_{\rho 1}(T)\right\rangle+\left\langle h_{\rho 2}^{\epsilon}, E G_{0 x}\left(y_{\rho}(0)\right) y_{\rho 1}(0)\right\rangle \\
& +\left\langle h_{\rho 0}^{\epsilon}, E \gamma_{y}\left(y_{\rho}(0)\right) y_{\rho 1}(0)\right\rangle+\epsilon \sqrt{\rho}+o(\epsilon) \geq 0 .
\end{aligned}
$$

Let $\left(p_{\rho}^{\epsilon}, k_{\rho}^{\epsilon}, q_{\rho}^{\epsilon}\right)$ be the solution of

$$
\left\{\begin{aligned}
-d p_{\rho}^{\epsilon} & =\left[f_{x}^{*}\left(x_{\rho}, v_{\rho}\right) p_{\rho}^{\epsilon}+g_{x}^{*}\left(x_{\rho}, y_{\rho}, z_{\rho}, v_{\rho}\right) q_{\rho}^{\epsilon}+\sigma_{x}^{*}\left(x_{\rho}\right) k_{\rho}^{\epsilon}\right] d t-k_{\rho}^{\epsilon} d W_{t}, \\
p_{\rho}^{\epsilon}(T) & =G_{1 x}\left(x_{\rho}(T)\right) h \rho_{1}^{\epsilon}-h_{x}^{*}\left(x_{\rho}(T)\right) q_{\rho}^{\epsilon}(T), \\
-d q_{\rho}^{\epsilon} & =g_{y}^{*}\left(x_{\rho}, y_{\rho}, z_{\rho}, v_{\rho}\right) q_{\rho}^{\epsilon} d t+g_{z}\left(x_{\rho}, y_{\rho}, z_{\rho}, v_{\rho}\right) q_{\rho}^{\epsilon} d W_{t}, \\
q_{\rho}^{\epsilon}(0) & =-\left(G_{0 x}\left(y_{\rho}(0)\right) h_{\rho 2}^{\epsilon}+\gamma_{y}\left(y_{\rho}(0)\right) h_{\rho 0}^{\epsilon}\right)
\end{aligned}\right.
$$

Using Itô's formula, (19) can be rewritten as

$$
\begin{aligned}
E \int_{0}^{T}[ & H\left(x_{\rho}, y_{\rho}, z_{\rho}, v_{\rho}^{\epsilon}, p_{\rho}^{\epsilon}, q_{\rho}^{\epsilon}, k_{\rho}^{\epsilon}\right) \\
& \left.-H\left(x_{\rho}, y_{\rho}, z_{\rho}, v_{\rho}, p_{\rho}^{\epsilon}, q_{\rho}^{\epsilon}, k_{\rho}^{\epsilon}\right)\right] d t+\epsilon \sqrt{\rho}+o(\epsilon) \geq 0,
\end{aligned}
$$


where

$$
H(x, y, z, v, p, q, k) \triangleq\langle p, f(x, v)\rangle+\langle q, g(x, y, z, v)\rangle+\langle k, \sigma(x)\rangle
$$

Since

$$
\lim _{\epsilon \rightarrow 0}\left(\left|h_{\rho 0}^{\epsilon}\right|^{2}+\left\|h_{\rho 1}^{\epsilon}\right\|^{2}+\left\|h_{\rho 2}^{\epsilon}\right\|^{2}\right)=1,
$$

there exists a convergent subsequence $\left(h_{\rho 0}^{\epsilon}, h_{\rho 1}^{\epsilon}, h_{\rho 2}^{\epsilon}\right)$ with

$$
\begin{aligned}
& \left(h_{\rho 0}^{\epsilon}, h_{\rho 1}^{\epsilon}, h_{\rho 2}^{\epsilon}\right) \rightarrow\left(h_{\rho 0}, h_{\rho 1}, h_{\rho 2}\right), \quad \epsilon \rightarrow 0, \\
& \left|h_{\rho 0}\right|^{2}+\left\|h_{\rho 1}\right\|^{2}+\left\|h_{\rho 2}\right\|^{2}=1 .
\end{aligned}
$$

Let $\left(p_{\rho}, q_{\rho}, k_{\rho}\right)$ be the solution of

$$
\left\{\begin{aligned}
-d p_{\rho} & =\left[f_{x}^{*}\left(x_{\rho}, v_{\rho}\right) p_{\rho}+g_{x}^{*}\left(x_{\rho}, y_{\rho}, z_{\rho}, v_{\rho}\right) q_{\rho}+\sigma_{x}^{*}\left(x_{\rho}\right) k_{\rho}\right] d t-k_{\rho} d W_{t} \\
p_{\rho}(T) & =G_{1 x}\left(x_{\rho}(T)\right) h_{\rho 1}-h_{x}^{*}\left(x_{\rho}(T)\right) q_{\rho}(T) \\
-d q_{\rho} & =g_{y}^{*}\left(x_{\rho}, y_{\rho}, z_{\rho}, v_{\rho}\right) q_{\rho} d t+g_{z}^{*}\left(x_{\rho}, y_{\rho}, z_{\rho}, v_{\rho}\right) q_{\rho} d W_{t} \\
q_{\rho}(0) & =-\left(G_{0 x}\left(y_{\rho}(0)\right) h_{\rho 2}+\gamma_{y}\left(y_{\rho}(0)\right) h_{\rho 0}\right)
\end{aligned}\right.
$$

It can be easily proved that

$$
\begin{array}{lll}
p_{\rho}^{\epsilon} \rightarrow p_{\rho} & \text { in } & \mathscr{L}_{\mathscr{F}}^{2}\left(0, T, R^{n}\right), \\
q_{\rho}^{\epsilon} \rightarrow q_{\rho} & \text { in } & \mathscr{L}_{F}^{2}\left(0, T, R^{m}\right), \\
k_{\rho}^{\epsilon} \rightarrow k_{\rho} & \text { in } & \mathscr{L}_{\mathscr{F}}^{2}\left(0, T, \mathscr{L}\left(R^{d}, R^{n}\right)\right) .
\end{array}
$$

So from (21) we have

$$
\begin{aligned}
& H\left(x_{\rho}, y_{\rho}, z_{\rho}, v, p_{\rho}, q_{\rho}, k_{\rho}\right) \\
- & H\left(x_{\rho}, y_{\rho}, z_{\rho}, v_{\rho}, p_{\rho}, q_{\rho}, k_{\rho}\right)+\sqrt{\rho} \geq 0 . \quad \forall v \in U, \quad \text { a.e., a.s. }
\end{aligned}
$$

Similarly from (22), there exists a subsequence of $\left(h_{\rho 0}, h_{\rho 1}, h_{\rho 2}\right)$ which converges to $\left(h_{0}, h_{1}, h_{2}\right)$ with

$$
\left|h_{0}\right|^{2}+\left\|h_{1}\right\|^{2}+\left\|h_{2}\right\|^{2}=1 \text {. }
$$

Since $v_{\rho}(\cdot) \rightarrow u(\cdot) \quad \rho \rightarrow 0$, consequently,

$$
\begin{array}{rll}
x_{\rho}(\cdot) \rightarrow x(\cdot) & \text { in } & \mathscr{L}_{\mathscr{F}}^{2}\left(0, T, R^{n}\right), \\
y_{\rho}(\cdot) \rightarrow y(\cdot) & \text { in } & \mathscr{L}_{F}^{2}\left(0, T, R^{m}\right), \\
z_{\rho}(\cdot) \rightarrow z(\cdot) & \text { in } & \mathscr{L}_{F}^{2}\left(0, T, \mathscr{L}\left(R^{d}, R^{n}\right)\right), \\
x_{\rho 1}(\cdot) \rightarrow x_{1}(\cdot) & \text { in } & \mathscr{L}_{F}^{2}\left(0, T, R^{n}\right), \\
y_{\rho 1}(\cdot) \rightarrow y_{1}(\cdot) & \text { in } & \mathscr{L}_{F}^{2}\left(0, T, R^{m}\right), \\
z_{\rho 1}(\cdot) \rightarrow z_{1}(\cdot) & \text { in } & \mathscr{L}_{F}^{2}\left(0, T, \mathscr{L}\left(R^{d}, R^{n}\right)\right),
\end{array}
$$


where $\left(x_{1}(\cdot), y_{1}(\cdot), z_{1}(\cdot)\right)$ is the solution of the variational equations whose forms are the same as (2).

We introduce the adjoint equations of the above variational equations as

$$
\left\{\begin{aligned}
-d p & =\left[f_{x}^{*}(x, u) p+g_{x}^{*}(x, y, z, u) q+\sigma_{x}^{*}(x) k\right] d t-k d W_{t} \\
p(T) & =G_{1 x}(x(T)) h_{1}-h_{x}^{*}(x(T)) q(T) \\
-d q & =g_{y}^{*}(x, y, z, u) q d t+g_{z}^{*}(x, y, z, u) q d W_{t} \\
q(0) & =-\left(G_{0 x}(y(0)) h_{2}+\gamma_{y}(y(0)) h_{0}\right)
\end{aligned}\right.
$$

It can be proved that

$$
\begin{array}{lll}
p_{\rho}(\cdot) \rightarrow p(\cdot) & \text { in } & \mathscr{L}_{\mathscr{F}}^{2}\left(0, T, R^{n}\right), \\
q_{\rho}(\cdot) \rightarrow q(\cdot) & \text { in } & \mathscr{L}_{\mathscr{F}}^{2}\left(0, T, R^{m}\right), \\
k_{\rho}(\cdot) \rightarrow k(\cdot) & \text { in } & \mathscr{L}_{\mathscr{F}}^{2}\left(0, T, \mathscr{L}\left(R^{d}, R^{n}\right)\right) .
\end{array}
$$

Let $\rho \rightarrow 0$ in (23). Then the following inequality holds.

$$
\begin{aligned}
& H(x(t), y(t), z(t), v, p(t), q(t), k(t))- \\
& H(x(t), y(t), z(t), u(t), p(t), q(t), k(t)) \geq 0, \quad \forall v \in U, \quad \text { a.e., a.s. }
\end{aligned}
$$

So we have the following theorem.

THEOREM 2. Assume $\left(H_{1}\right),\left(H_{2}\right),\left(H_{3}\right)$ and $\left(H_{4}\right)$ hold. Let $(u(\cdot), x(\cdot), y(\cdot), z(\cdot))$ be an optimal solution of the optimal control problem stated at the beginning of this section, and $(p(\cdot), q(\cdot), k(\cdot))$ be the corresponding solution of the adjoint equations (24). Then the maximum principle (25) holds.

\section{Remark}

For the forward stochastic system in which control enters into the diffusion coefficient, the maximum principle in global form can be found in Arkin and Saksonov [4], Bismut [6], Cadenillas and Karatzas [7], and Peng [12]. But for the forward and backward stochastic system, such an optimal control problem is still an open problem.

\section{Acknowledgements}

This work was partially supported by the Chinese Natural Science Foundation. 


\section{References}

[1] N. U. Ahmed, "Existence of optimal controls for a class of systems governed by differential inclusions on a Banach space", J. Optim. Theory Appl. 50 (1986) 213-237.

[2] N. U. Ahmed, "Relaxed controls for stochastic boundary value problems in finite dimension", in Lecture notes in control and information science 149, (Springer, Berlin, 1991).

[3] V. Arkin and M. Saksonov, "Necessary optimality conditions for stochastic differential equations", Soviet Math. Dokl. 20 (1973) 1-5.

[4] V. Arkin and M. Saksonov, "On the theory of the stochastic maximum principle in continuous time problems", in Models and methods of stochastic optimization, (Central Economics and Mathematical Institute-Academy of Sciences of the USSR, 1983) 3-26.

[5] A. Bensoussam, "Lectures on stochastic control", in Lecture notes in mathematics, 972, (SpringerVerlag, Berlin).

[6] J. M. Bismut, "Conjugate convex functions in optimal stochastic control", J. Math. Anal. Appl. 44 (1973) 384-404.

[7] A. Cadenillas and I. Karatzas, "The stochastic maximum principle for linear convex optimal control with random coefficients", SIAM J. Control Optim. (to appear).

[8] R. J. Elliott, "The optimal control of diffusions", Appl. Math. Optim. 22 (1990) 229-240.

[9] N. Ikeda and S. Watanabe, Stochastic differential equations and diffusion processes (North Holland Publishing Company, 1981).

[10] H. J. Kushner, "Necessary conditions for continuous parameter stochastic optimization problems", SIAM J. Control 10 (1972) 550-565.

[11] E. Pardoux and S. Peng, "Adapted solution of backward stochastic equation", Systems and Control Letters 14 (1990) 55-61.

[12] S. Peng, “A general stochastic maximum principle for optimal control problems", SIAM J. Cont. 28 (1990) 966-979.

[13] S. Peng, "Backward stochastic differential equation and application to optimal control", Appl. Math. Optim. 27 (1993) 125-144. 